\title{
No-reference Sharpness Index for Scanning Electron Microscopy I mages Based on Dark Channel Prior
}

\author{
Qiaoyue $\mathrm{Li}^{1}$, Leida $\mathrm{Li}^{1}$, Zhaolin $\mathrm{Lu}^{1,{ }^{1,}}$, Yu Zhou ${ }^{1}$, Hancheng $\mathrm{Zhu}^{1}$ \\ ${ }^{1}$ School of Information and Control Engineering, China University of Mining and Technology \\ Xuzhou, Jiangsu 221116 - China \\ [e-mail: zhaolinlu@cumt.edu.cn] \\ *Corresponding author: Zhaolin Lu
}

Received August 2, 2018; revised October 20, 2018; accepted December 4, 2018;

published May 31, 2019

\begin{abstract}
Scanning electron microscopy (SEM) image can link with the microscopic world through reflecting interaction between electrons and materials. The SEM images are easily subject to blurring distortions during the imaging process. Inspired by the fact that dark channel prior captures the changes to blurred SEM images caused by the blur process, we propose a method to evaluate the SEM images sharpness based on the dark channel prior. A SEM image database is first established with mean opinion score collected as ground truth. For the quality assessment of the SEM image, the dark channel map is generated. Since blurring is typically characterized by the spread of edge, edge of dark channel map is extracted. Then noise is removed by an edge-preserving filter. Finally, the maximum gradient and the average gradient of image are combined to generate the final sharpness score. The experimental results on the SEM blurred image database show that the proposed algorithm outperforms both the existing state-of-the-art image sharpness metrics and the general-purpose no-reference quality metrics.
\end{abstract}

Keywords: Image quality assessment, No-reference, Sharpness, Dark channel, Scanning electron microscopy 


\section{Introduction}

Scanning electron microscopy (SEM) has been widely applied to display the microstructures in many fields, such as medicinal chemistry, medical science and genetics, etc. Typically, various types of distortions directly affect the quality of the SEM images during the process of SEM imaging [1-2], e.g. blurring, noise, astigmatism, contrast and brightness. Among them, blurring is one of the most common distortions that affects the quality perception. Therefore, the sharpness assessment of the SEM images is of great significance. Image quality assessment (IQA) contains subjective evaluation and objective evaluation. The former is the most accurate method because human is the ultimate receiver of images. However, the subjective evaluation is time-consuming. For objective IQA, it can be divided into full reference (FR) [3-5], reduced reference (RR) [6-8] and no reference (NR) [9-13]. FR methods need the complete information of the original images. RR methods require parts of the original information, and NR methods operate without any reference information.

Since the reference information of images are difficult to obtain, NR methods are highly desired. NR methods can be classified into two categories according to the type of distortion. The first category is designed for the specific type of distortion. As aforementioned, blurring is the most common distortion, so only the sharpness assessment approaches are summarized as follows. Ferzli et al. [14] proposed the just noticeable blur (JNB) algorithm. Through the combination of the concept of JNB with the probability sum model, the algorithm was used to predict the relative amount of the blurred image with different contents. In [15], Bahrami et al. defined the maximum local variation (MLV) for each pixel as the maximum intensity difference between the pixel and its 8-neighborhood, and the standard deviation of the MLV distribution was defined as the sharpness score. In [16], Li et al. proposed a blind image blur evaluation (BIBLE) algorithm that the utilization of discrete Tchebichef moments for measuring the variation of image shape caused by image blur distortion. The second category of NR quality metrics are for general distortions. In [17], Saad et al. used the Bayesian inference mode to predict the quality of image. The feature was based on natural scene statistical model of image discrete cosine transform coefficients. Zhang et al. [18] proposed a quality evaluation algorithm using log-derivative statistics of natural scenes. Statistical features related to image quality were extracted at two image scales in both spatial and frequency domain. Liu et al. [19] proposed a spatial-spectral entropy-based quality (SSEQ) index model to predict the quality of the image. Although these algorithms have achieved great advances on natural images, they can not effectively evaluate the quality of SEM images, which are different from natural images.

The SEM images are grayscale images, they look gloomy on the background and show the surfaces of objects. So the content on SEM image can give rise to low intensities in the dark channel [20]. In addition, it is found that dark channel prior is sensitive to the changes to blurred images caused by the blur process [21]. The blurred images have fewer dark channel and different blurred images have obvious differences in the dark channel maps. Based on these observations, we propose a NR sharpness index for SEM images based on dark channel prior. Due to the lack of SEM image database, we first build a database and collect subjective scores of SEM images through user study. Because blurred image is typically characterized by the spread of edges, the edge of dark channel maps are extracted. Then an edge-preserving operator is employed to remove noise effect but preserve the edge information based on the weighted least squares (WLS) framework. Finally, the maximum gradient and the average gradient are combined to generate the sharpness score of the SEM 
image. The experimental results on the SEM blurred image database demonstrate that the proposed method outperforms the state-of-the-art metrics.

\section{Database establishment and subjective experiment}

Image databases play an important role in understanding how people perceive the quality of images, and they are crucial for designing and evaluating IQA models in terms of the consistency with human subjective evaluations. For SEM, the quality evaluation algorithm which can guide the selection of imaging parameters is highly desired. To the best of our knowledge, such a database for SEM images is still lacking. Therefore, we need to build a database to test the performance of the methods. In this section, we build a SEM database, followed by a subjective test to collect quality scores.

\subsection{Database establishment}

All SEM images were taken using a scanning electron microscope from the Advanced Analysis and Computation Center in China University of Mining and Technology. The SEM image database contains 50 groups of images with diversified contents, including ants, metals, coal particles, colloids, etc. Fig. 1 shows eight clear SEM images of different substances. For each image, there are 3 blurred distorted versions, the size of the image is $1024 * 884$, a total of 150 images. The blurring degrees of SEM images are related to scanning time. We can get SEM images with different blurring degrees by adjusting different scanning time.

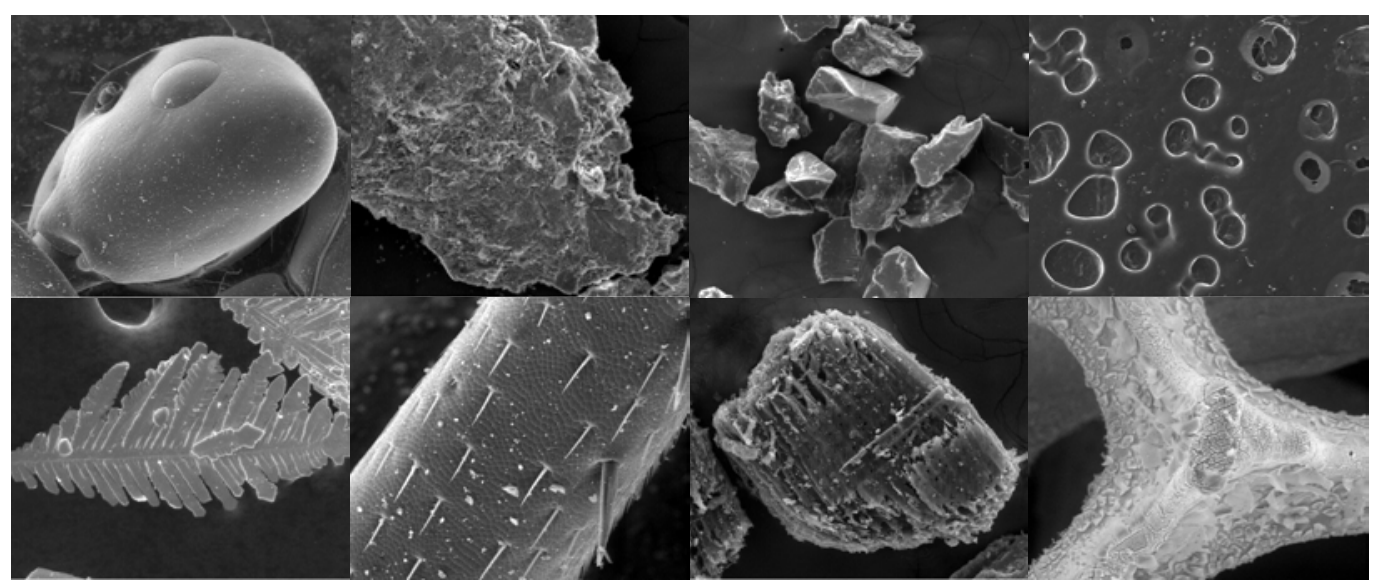

Fig. 1. Clear SEM images of 8 different substances

\subsection{Subjective experiment}

The subjective experiment is conducted using a PC of Intel (R) Pentium (R) CPU G645 2.9GHz. The image is displayed on a LCD with a resolution of $1920 * 1080$. The experiment is conducted in a normal light indoor workplace. Thirty subjects with no knowledge of image processing participate in the subjective experiment. Besides, an interactive platform is designed to display the test images. Meanwhile, subjects also learned the principles of platform operation and criterion of image quality rating. The perceive image quality of the experiment is divided into five levels [22], corresponding to consecutive 1-5 points, where 5 is the best and the 1 is the worst. The subjects click to select the perceived image quality score. Thirty subjects rate all 650 images. After completing the subjective experiment, each 
test image has 30 subjective scores from 30 subjects. In order to reduce the error of experimental results, five outliers are removed from 30 ratings for each image, leaving only 25 valid ratings. Meanwhile, the average value of the 25 valid data represents the subjective experimental result of the image. We use 150 blurred images and the corresponding mean opinion score (MOS) values to prepare further study. Fig. 2 shows an original image and the corresponding images with different levels of blur.

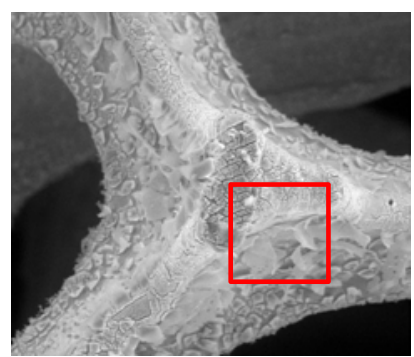

(a) $\mathrm{MOS}=5.0000$

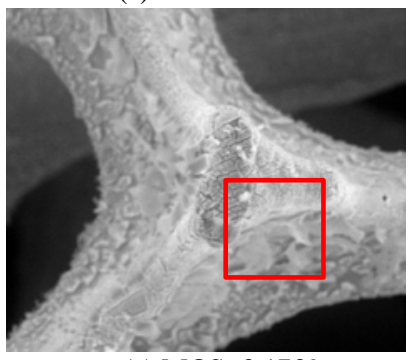

(c) $\mathrm{MOS}=3.1739$
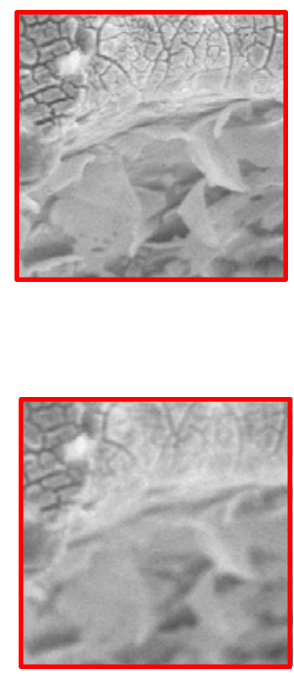

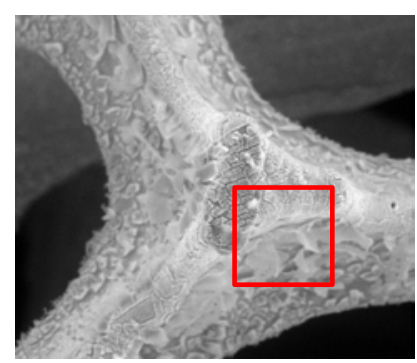

(b) $\mathrm{MOS}=4.3200$

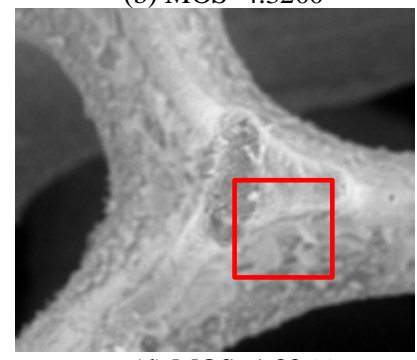

(d) $\mathrm{MOS}=1.8366$
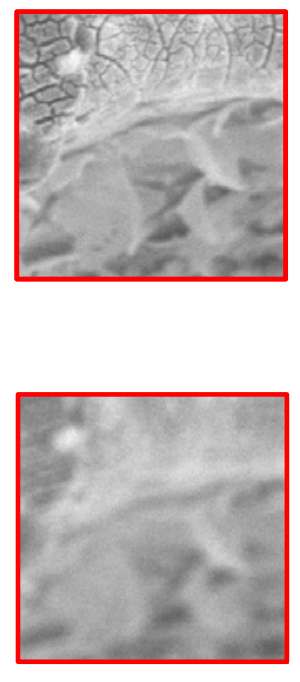

Fig. 2. The original SEM image and the corresponding images with different levels of blurring: (a) MOS=5.0000; (b) MOS=4.3200; (c) MOS=3.1739; (d) MOS=1.8366.

\section{Proposed SEM image sharpness metric}

Due to the very narrow electron beam, SEM images have a large depth of field producing a characteristic of three-dimensional appearance for understanding the surface structure of a sample. According to the characteristics of SEM images described above and the ability of dark channel prior to captures the changes to blurred images [21], the dark channel maps are first generated. The study has shown that the human visual system has multi-channel characteristics [23]. Each layer of images owns individual features, and high frequency part mainly provides the edge and contour information of the image. The essence of image blurring is that the high frequency component of the image is attenuated. Therefore, the image edge can reflect the blurring degree. Based on the above points, the edge of dark channel maps are extracted. Then an edge-preserving smoothing filter is used to remove noise while enhancing the edge information based on the weighted least squares (WLS). According to the characteristics of the human visual system, the maximum gradient and the average gradient are integrated to generate the final quality score of the SEM images. Fig. 3 shows the flowchart of the proposed metric. 


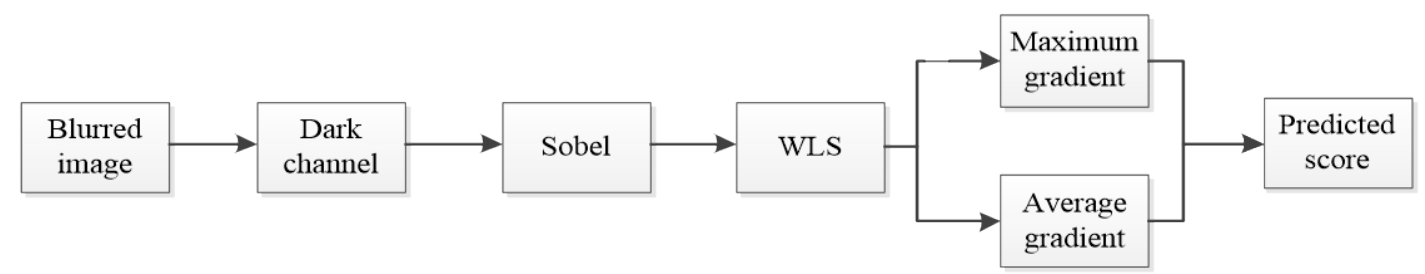

Fig. 3. The flowchart of the proposed metric

\subsection{Dark channel}

SEM is the interaction of electrons and material to obtain the surface morphology of the sample. Pan [21] et al. found that some pixels that in clean image are not dark when averaged with neighboring high intensity pixels during the blur process. And the dark channel [20] maps are different with different blur degrees of SEM images.

For an image $I$, the dark channel is defined as:

$$
D(I)(x)=\min _{y \in \Omega(x)}\left(\min _{c \in\{r, g, b\}} I^{c}(y)\right),
$$

where $x$ and $y$ are the positions of the pixels and $\Omega(x)$ is the center of the image block at $x$, which $I^{c}$ is the $c$-th color channel. As the SEM images are grayscale images, the equation [21] can be simplified as:

$$
D(I)(x)=\min _{y \in \Omega(x)}(I(y))
$$

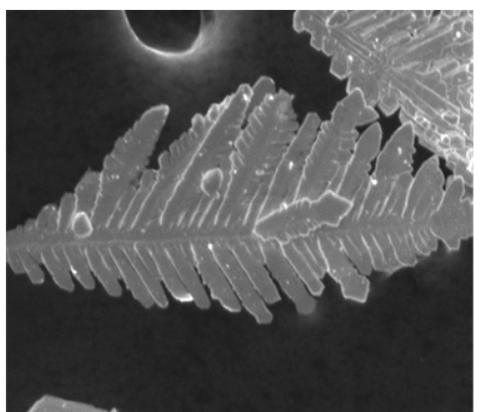

(a) Original blurred image

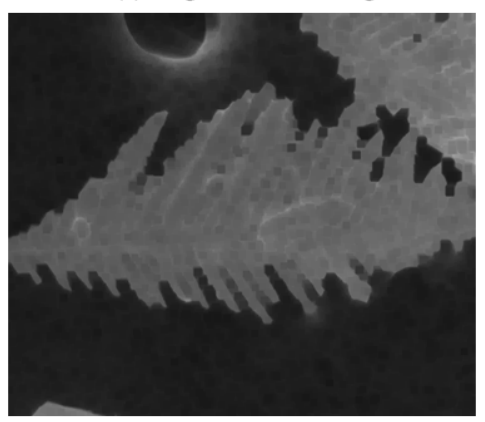

(c) $15 * 15$

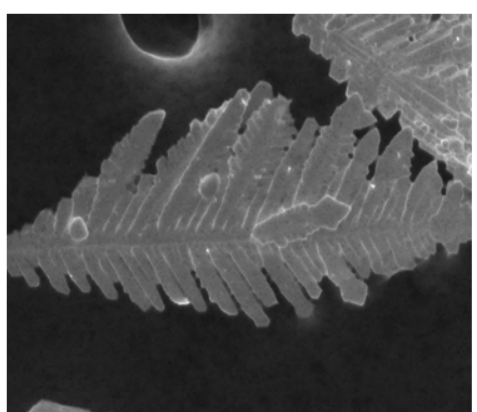

(b) 5 *5

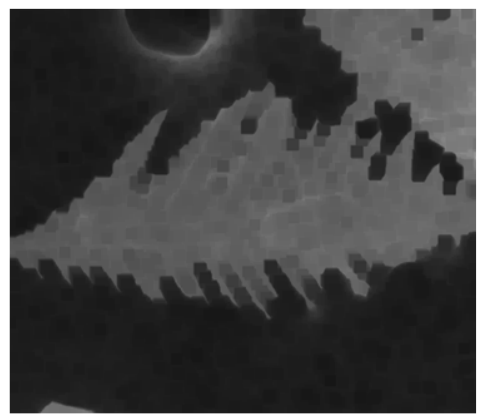

(d) $24 * 24$

Fig. 4. The original blurred image and dark channel maps with three different block sizes 
Fig. 4 shows the original blurred image and the dark channel maps calculated using three different block sizes. For the small block size, the image is not changed in comparison with the original image. By contrast, too big block size tends to generated dark channel maps with much less information maintained. In this work, the size of image block is empirically set to $15 * 15$.

\subsection{Edge detection}

In the process of image quality evaluation, edge features can be used to improve the accuracy of image quality evaluation. Therefore, the method of extracting edge features for SEM image quality evaluation can greatly reduce the amount of calculation and improve the efficiency of calculation. In addition, when the image distortion is heavy, the edge feature is more easily detected. Fig. 5 shows different blur levels of the SEM images and Fig. 6 shows dark channel maps that the $15 * 15$ block size of three blur degrees. Through observation from Fig. 6, it can be seen that the texture is simple and the edge is more prominent.
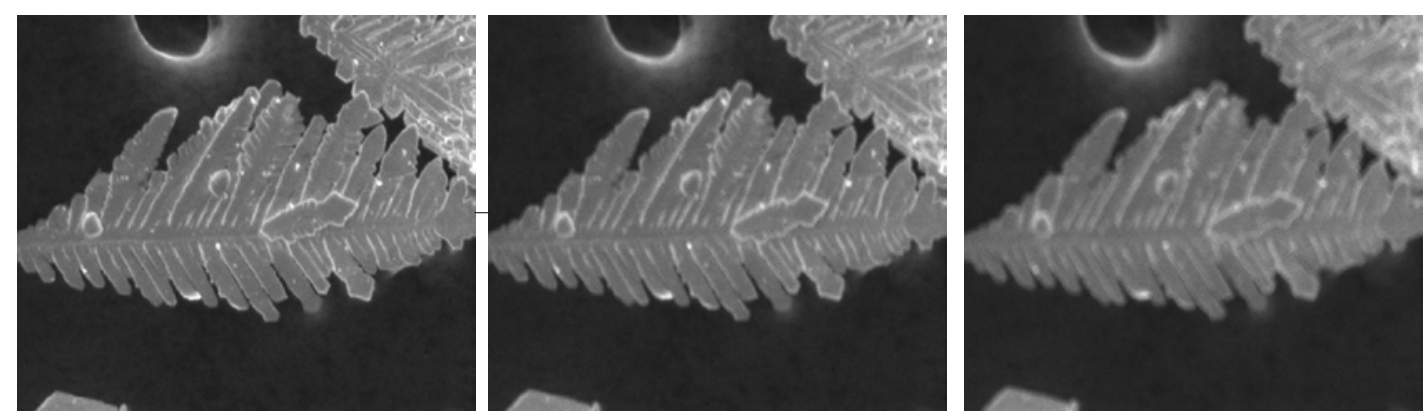

Fig. 5. Different blur levels of the SEM images
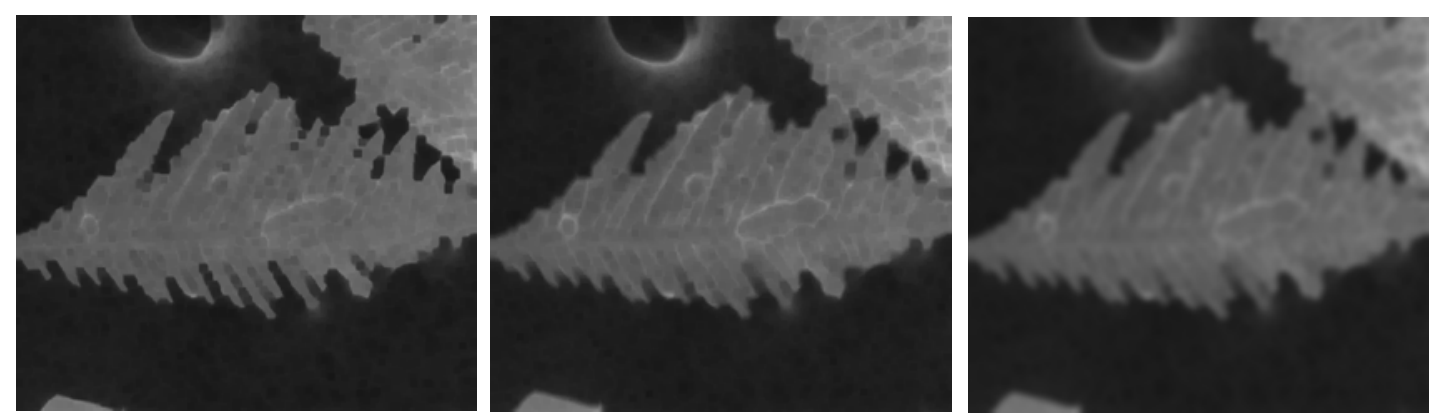

Fig. 6. Dark channel maps that the $15 * 15$ block size of three blur degrees

After dark channel processing, the Sobel edge operator is used to filter the horizontal and vertical direction of the image, producing the edge map. 
The Sobel operator is defined as:

$$
G=\left|G_{x}\right|+\left|G_{y}\right|
$$

where $G_{x}$ and $G_{y}$ are defined as:

$$
\begin{gathered}
G_{x}=\left[\begin{array}{ccc}
-1 & 0 & 1 \\
-2 & 0 & 2 \\
-1 & 0 & 1
\end{array}\right] * I \\
G_{y}=\left[\begin{array}{ccc}
1 & 2 & 1 \\
0 & 0 & 0 \\
-1 & -2 & -1
\end{array}\right] * I
\end{gathered}
$$

\subsection{Edge preserving smoothing filter based on weighted least squares}

The Weighted Least Squares Framework (WLS) [24] is a non-linear, edge-preserving smoothing method that captures the details of various scales by multi-scale edge-preserving decomposition. The edge preserving smoothing filter based on weighted least squares is used to remove the noise while maintaining the strength of the edge.

For one input images $G$, on the one hand, we want the target image $U$ to be as close as possible to $G$, while $U$ should be as smooth as possible except for some places where the edge gradient changes a lot in $G$. Formally, we have:

$$
F_{\lambda}(g)=\left(I+\lambda L_{g}\right) U=G,
$$

where $L_{g}=D_{x}^{T} B_{x} D_{x}+D_{y}^{T} B_{y} D_{y} . \quad D_{x}$ and $D_{y}$ are discrete differentiation operators. $B_{x}$ and $B_{y}$ are smoothness weights, the smoothness is required to be performed in a spatially varying manner which depending on $U . \lambda$ is the balance factor between the data item and the smoothed item. $\lambda$ controls the smoothness and increases the value of the result in the progressive smoothed image.

Fig. 7 shows the image edge of different blurring degree after dark channel processing. It can be seen from the figure that there are much noise in the image, which seriously affects the quality of the image. Fig. 8 shows the images after the edge-preserving filtering. It can be seen from Fig. 7 and Fig. 8 that the noise is weakened and the edge information remains intact. 

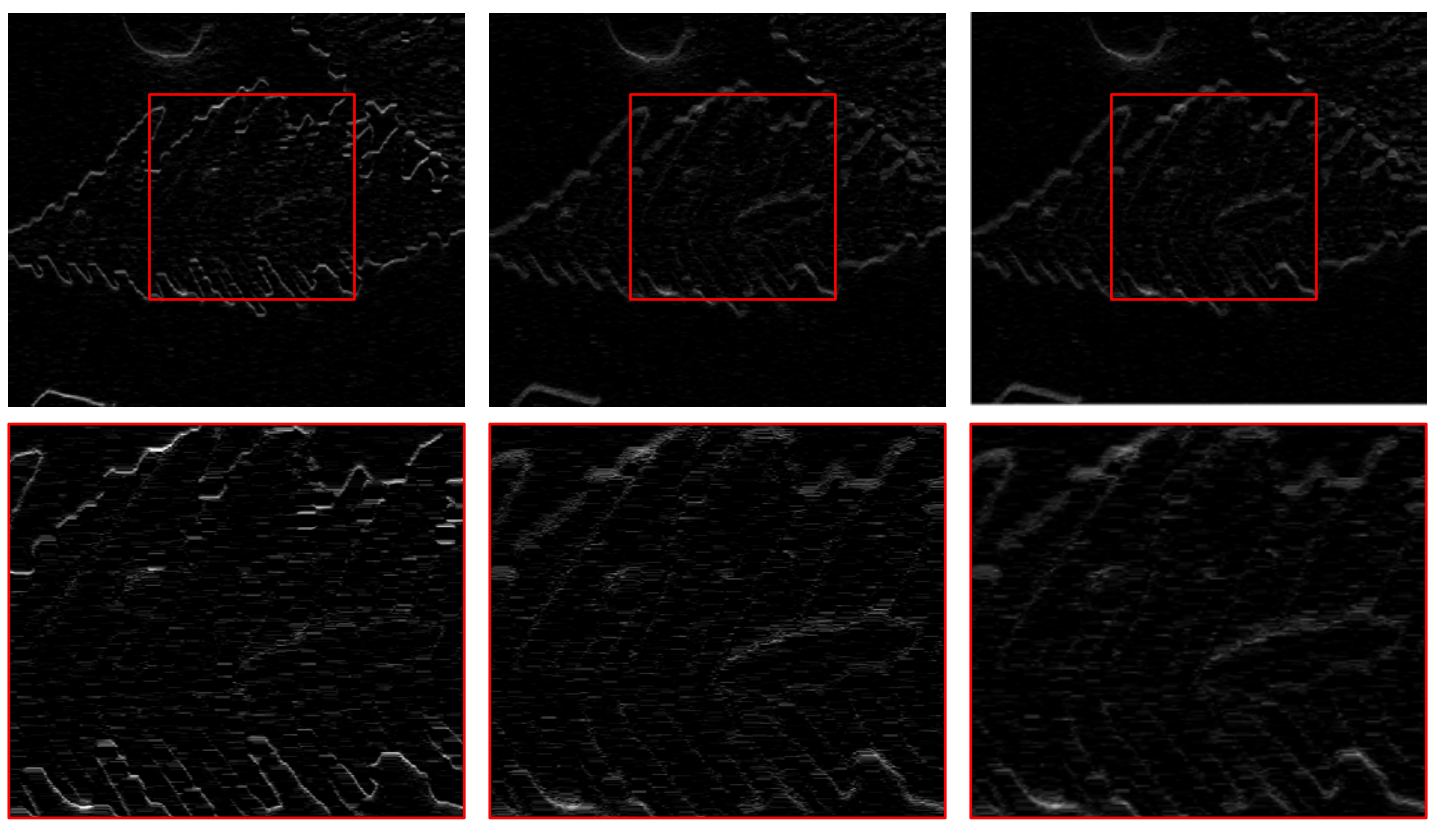

Fig. 7. The image edge of different blurring degree after dark channel processing
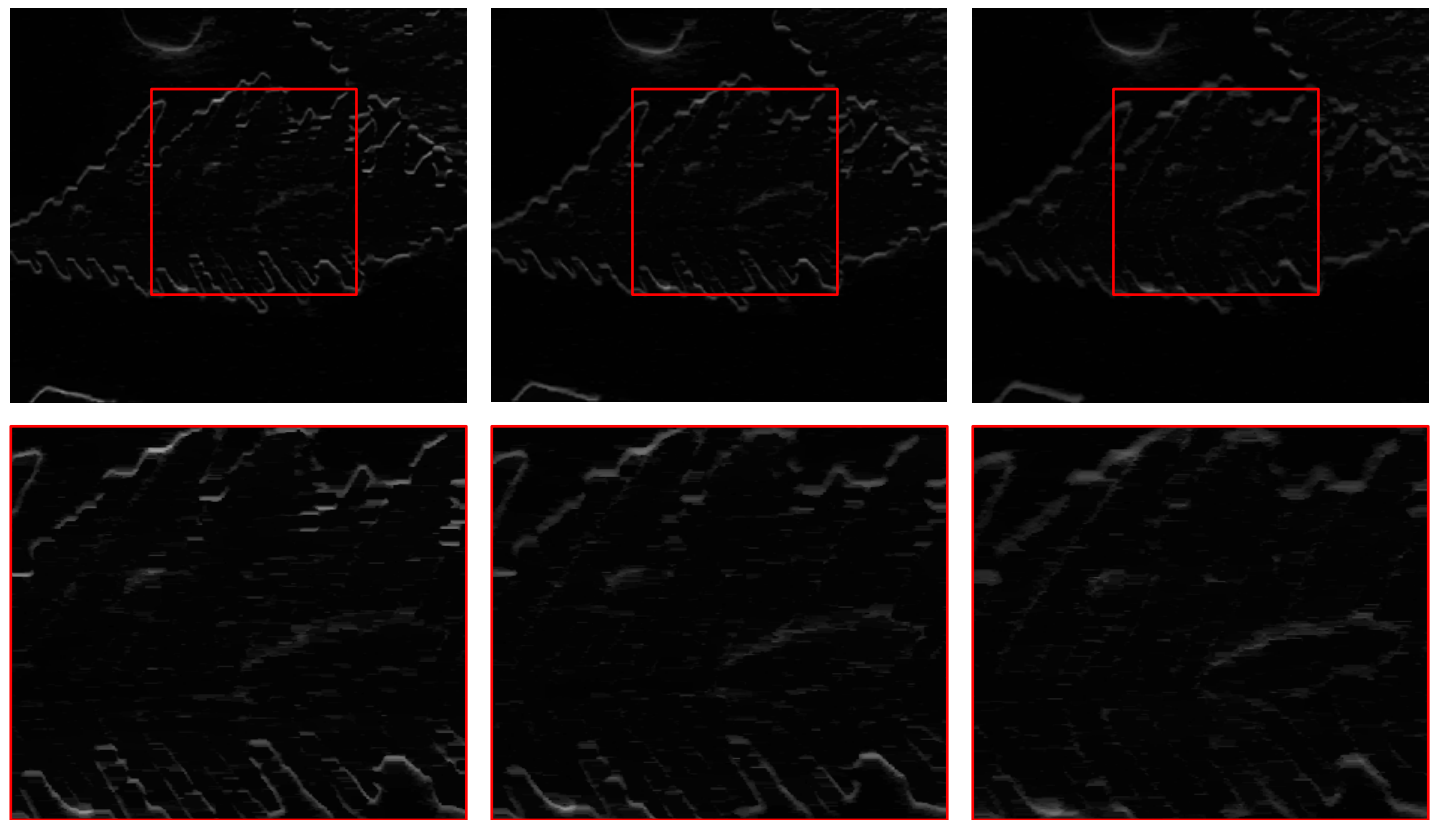

Fig. 8. The images after the edge-preserving filter

\subsection{Quality score}

Zhan et al. [25] found that the maximum gradient can be effective for image sharpness assessment. And the average gradient reflects the sharpness of the global content of the image. To eliminate the influence of the image content, the maximum gradient and the average gradient are combined to generate the final sharpness score. 
The maximum gradient and the average gradient of the image are defined as:

$$
\begin{gathered}
M G=\max (G(i, j)), \\
A G=\left(\sum_{i, j} G(i, j) /(w \times h)\right),
\end{gathered}
$$

where $M G$ and $A G$ are the maximum gradient and the average gradient respectively, and $w$ and $h$ are the size of the image.

Finally, the SEM image sharpness score is defined as:

$$
I Q A=M G \times A G^{-\alpha},
$$

In this work, $\alpha$ is empirically set to 0.4366 .

\section{Experimental results and analysis}

\subsection{Experiment Settings}

To evaluate the performance of the proposed method, we adopt three commonly used criteria, including Pearson linear correlation coefficients (PLCC), Spearman rank order correlation coefficients (SRCC) and root mean square error (RMSE) [26]. PLCC and RMSE measure the accuracy of algorithm prediction, while SRCC is used to measure the prediction monotonicity. The objective quality scores and subjective scores are mapped to the same scale [27] by nonlinear fitting function:

$$
f(v)=\tau_{1}\left(\frac{1}{2}-\frac{1}{1+e^{\tau_{2}\left(v-\tau_{3}\right)}}\right)+\tau_{4} v+\tau_{5}
$$

where $\tau_{i}(i=1,2, \ldots 5)$ are the fitted parameters.

\subsection{Performance Evaluation}

1) Comparison with NR-IQA sharpness metrics: In this part, we compare the performance of the proposed method with eleven existing no-reference image sharpness metrics on the SEM blurred image database, which are JNB [14], CPBD [28], Marz. [29], LPC [30], MLV [15], BIBLE [16], S3 [31], SPARISH [32], FISH [33], ARISM [34], SVC [35]. Table 1 summarizes the experimental results, where the best performance values are marked in boldface.

Table 1. Performance of the proposed method and state-of-the-art sharpness metrics

\begin{tabular}{cccc}
\hline Algorithms & PLCC & SRCC & RMSE \\
\hline LPC [30] & 0.7531 & 0.6828 & 0.4552 \\
MLV [15] & 0.7155 & 0.6931 & 0.4833 \\
BIBLE [16] & 0.4244 & 0.4077 & 0.6264 \\
S3 [31] & 0.3919 & 0.3610 & 0.6365 \\
JNB [14] & 0.3210 & 0.2869 & 0.6552 \\
SPARISH [32] & 0.2942 & 0.2513 & 0.6612
\end{tabular}




\begin{tabular}{cccc} 
SVC [35] & 0.2919 & 0.2640 & 0.6617 \\
Marz. [29] & 0.2680 & 0.2446 & 0.6665 \\
FISH [33] & 0.2435 & 0.2194 & 0.6710 \\
CPBD [28] & 0.1399 & 0.6850 & 0.4466 \\
ARISM [34] & 0.0960 & 0.0756 & 0.6886 \\
Proposed & $\mathbf{0 . 8 3 9 1}$ & $\mathbf{0 . 8 2 8 6}$ & $\mathbf{0 . 3 7 6 4}$ \\
\hline
\end{tabular}

It can be seen from Table 1, the performances of proposed algorithm is better than other existing sharpness/blur algorithms in terms of monotonicity and prediction accuracy. This proves that SEM images are unnatural images. The quality evaluation methods based on natural images cannot evaluate the quality of SEM images very well. Therefore, the method of this paper which is designed for the characteristics of SEM images has achieved the best performance.

2) Comparison with general-purpose NR-IQA metrics: Several common NR image quality metrics proposed in the previous literature can predict the image quality without knowing the specific distortion type. So we further compare the proposed algorithm with the state-of-the-art general-purpose NR quality models on the SEM blurred image database, including BRISQUE [36], DESIQUE [18], BIQI [37], DIIVINE [38], BLLINDS-II [17], NIQE [39], SSEQ [19], QAC [40]. Table 2 summarizes the experimental results, where the best performance values are marked in boldface.

It can be seen from Table 2 that no matter the prediction accuracy or monotonicity, the proposed algorithm achieves the best performance among all compared algorithms. This also proves that the SEM images are unnatural images, and the general methods based on natural images are not suitable for SEM images.

Table 2. Proposed method and the state-of-the-art general algorithms

\begin{tabular}{cccc}
\hline Algorithms & PLCC & SRCC & RMSE \\
\hline BRISQUE [36] & 0.8276 & 0.8102 & 0.3812 \\
DESIQUE [18] & 0.8247 & 0.8059 & 0.3843 \\
BIQI [37] & 0.8127 & 0.7867 & 0.3983 \\
DIIVINE [38] & 0.7766 & 0.7461 & 0.4276 \\
BLIINDS-II [17] & 0.7698 & 0.7442 & 0.4320 \\
NIQE [39] & 0.7391 & 0.7311 & 0.4660 \\
SSEQ [19] & 0.6398 & 0.6323 & 0.5141 \\
QAC [40] & 0.2513 & 0.1616 & 0.6696 \\
Proposed & $\mathbf{0 . 8 3 9 1}$ & $\mathbf{0 . 8 2 8 6}$ & $\mathbf{0 . 3 7 6 4}$ \\
\hline
\end{tabular}

3) In order to compare the statistical significance of the proposed method and the existing metrics, we further conduct the F-test [41]. Fig. 9 shows the F statistics of the compared metrics against the proposed method. During the experiment, 95\% confidence level is used for the F-test. Table 3 and Table 4 are the statistical performance results of the sharpness image quality metrics and the general-purpose image metrics with the proposed method, 
respectively. The value of 1 indicates that the proposed method performs statistically better than other methods, and 0 indicate that the performance of the proposed method is statistically equivalent to the other methods. It can be seen from the table that the performance of proposed method is obviously superior to all the no-reference sharpness indicators and the five general-purpose NR quality metrics. The remaining three general-purpose NR metrics are statistically equivalent to the performance of this method.

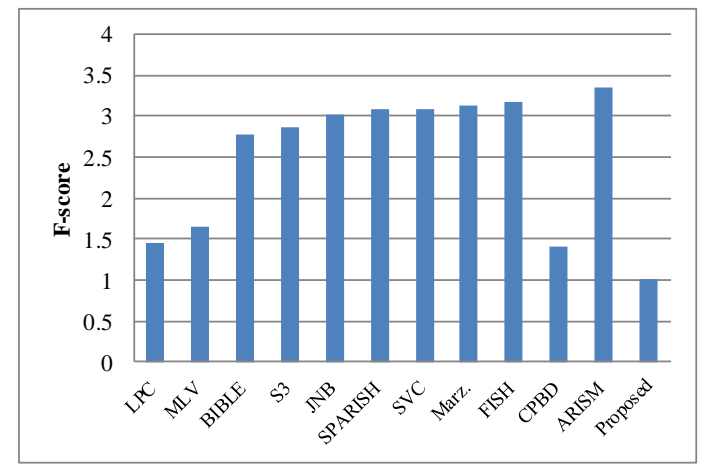

(a)

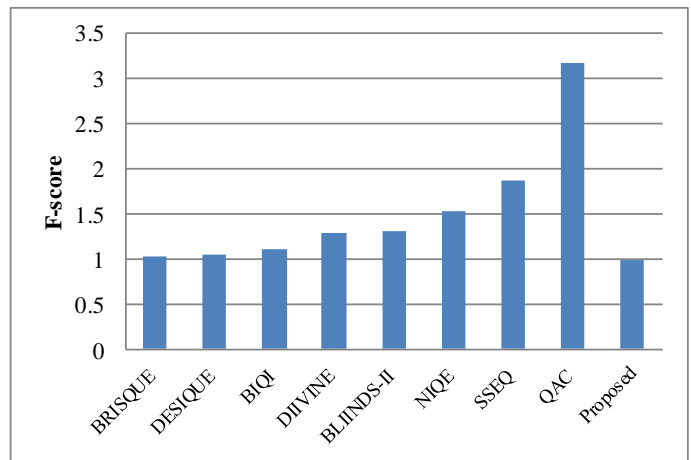

(b)

Fig. 9. The F statistics of the compared metrics against proposed method. (a) NR-IQA sharpness metrics with proposed metrics; (b) General-purpose NR-IQA metrics with proposed metrics.

Table 3. Statistical performance results of the sharpness image quality metrics with proposed method

\begin{tabular}{cccccccccccc}
\hline Metric & LPC & MLV & BIBLE & S3 & JNB & SPARISH & SVC & Marz. & FISH & CPBD & ARISM \\
\hline SEM & 1 & 1 & 1 & 1 & 1 & 1 & 1 & 1 & 1 & 1 & 1 \\
\hline
\end{tabular}

Table 4. Statistical performance results of the general-purpose image metrics with proposed method

\begin{tabular}{ccccccccc}
\hline Metric & BRISQUE & DESIQUE & BIQI & DIIVINE & $\begin{array}{c}\text { BLLINDS- } \\
\text { II }\end{array}$ & NIQE & SSEQ & QAC \\
\hline SEM & 0 & 0 & 0 & 1 & 1 & 1 & 1 & 1 \\
\hline
\end{tabular}

\subsection{Impact of dark channel block sizes}

In order to evaluate the impact of dark channel block sizes on the performance, we have tested different block sizes, ranging from $3 * 3$ to $27 * 27$. Table 5 lists the results of PLCC, SRCC and RMSE with different block sizes. It can be seen from the table that the performance varies with the sizes of the block. Moreover, we can see that the best results are obtained when the block size is $15 * 15$. Therefore, $15 * 15$ block size is used in this paper.

Table 5. The results of PLCC, SRCC and RMSE with different block sizes

\begin{tabular}{cccccccccc}
\hline Size & $\mathbf{3 x 3}$ & $\mathbf{6 x 6}$ & $\mathbf{9 x 9}$ & $\mathbf{1 2 x 1 2}$ & $\mathbf{1 5 x 1 5}$ & $\mathbf{1 8 x 1 8}$ & $\mathbf{2 1 x 2 1}$ & $\mathbf{2 4 x 2 4}$ & $\mathbf{2 7 x 2 7}$ \\
\hline PLCC & 0.7034 & 0.7974 & 0.7985 & 0.8181 & $\mathbf{0 . 8 3 9 1}$ & 0.8061 & 0.7794 & 0.7785 & 0.7644 \\
SRCC & 0.6918 & 0.7955 & 0.7966 & 0.8136 & $\mathbf{0 . 8 2 8 6}$ & 0.7961 & 0.7798 & 0.7768 & 0.7701 \\
RMSE & 0.4918 & 0.4175 & 0.4165 & 0.3980 & $\mathbf{0 . 3 7 6 4}$ & 0.4095 & 0.4334 & 0.4343 & 0.4460 \\
\hline
\end{tabular}




\section{Conclusion}

In this paper, due to the dark channel prior captures the changes to blurred images, we have proposed a no-reference sharpness index of SEM images based on dark channel prior. The SEM image database was built and a subjective experiment has been done to collect the ground truth of subjective scores. Experimental results on SEM blurred image database have shown that the proposed method is superior to the existing state-of-the-art algorithms. Although the proposed method has achieved notable improvement, it has the following limitations. First, the database size is limited, which needs to be enlarged. Second, it is specifically designed for the sharpness evaluation of the SEM images. However, in practice, the distortions introduced to the SEM images are diverse. That is to say the SEM image may be multiply-distorted. Therefore, designing a quality metric that is effective for the evaluation of the multiply-distorted SEM images is desirable. In the future, we will dedicate to not only constructing a large database but also designing the effective metric for the SEM images with multiple distortions.

\section{Acknowledgment}

This work is supported by the Fundamental Research Funds for the Central Universities under Grant 2017XKQY084.

\section{References}

[1] Z. Ruan, S. F. Mao, P. Zhang, H. M Li and Z. J. Ding, "Monte Carlo simulation of realistic beam-sample interaction in SEM: application to evaluation of sharpness measurement methods," in Proc. of SPIE Defense, Security, and Sensing, vol. 8729, pp.87290J, 2013.

Article (CrossRef Link).

[2] M. Lee, J. Cantone, J. Xu, L. Sun and R. Kim, "Improving SEM image quality using pixel super resolution technique," in Proc. of SPIE - The International Society for Optical Engineering, vol. 9050, no. 15, pp. 90500U-90500U-8, 2014. Article (CrossRef Link).

[3] Z. Wang, A. C. Bovik, H. R. Sheikh, and E.P. Simoncelli, "Image quality assessment: From error visibility to structural similarity," IEEE Transactions on Image Processing, vol. 13, no. 4, pp. 600-612, 2004. Article (CrossRef Link).

[4] H. R. Sheikh, and A. C. Bovik, "Image information and visual quality," IEEE Transactions on Image Processing, vol. 15, no. 2, pp. 430-444, 2006. Article (CrossRef Link).

[5] D. M. Chandler and S. S. Hemami, "VSNR: A wavelet based visual signal-to-noise ratio for natural images," IEEE Transactions on Image Processing, vol. 16, no. 9, pp. 2284-2298, 2007. Article (CrossRef Link).

[6] J. J. Wu, W. S. Lin, G. M. Shi, L. D. Li, and Y. M. Fang, "Orientation selectivity based visual pattern for reduced-reference image quality assessment," Information Sciences, vol. 351, pp. 18-29, 2016. Article (CrossRef Link).

[7] Z. Wang, and E. P. Simoncelli, "Reduced-reference image quality assessment using a wavelet-domain natural image statistic model,” in Proc. of SPIE, vol. 5666, pp. 149-159, 2005. Article (CrossRef Link).

[8] J. J. Wu, W. S. Lin, G. M. Shi and A. M. Liu, "Reduced-reference image quality assessment with visual information fidelity," IEEE Transactions on Multimedia, vol. 15, no. 7 pp. 1700-1705, 2013. Article (CrossRef Link).

[9] L. D. Li, H. C. Zhu, G. B. Yang, and J. S. Qian, "Referenceless Measure of Blocking Artifacts by Tchebichef Kernel Analysis,” IEEE Signal Processing Letters, vol. 21, no. 1, pp. 122-125, 2014. Article (CrossRef Link). 
[10] L. D. Li, Y. Zhou, W. S. Lin, J. J. Wu, X. F. Zhang, and B. J. Chen, "No-reference quality assessment of deblocked images,” Neurocomputing, vol. 177, no. C, pp. 572-584, 2016. Article (CrossRef Link).

[11] K. Gu, S. Q. Wang, H. Yang, W. S. Lin, G. T. Zhai, X. K. Yang and W. J. Zhang, "Saliency-guided quality assessment of screen content images," IEEE Transactions on Multimedia, vol. 18, no. 6, pp. 1098-1110, 2016. Article (CrossRef Link).

[12] L. D. Li, Y. Yan, Y. M. Fang, S. Q. Wang, L. Tang, and J. S. Qian, "Perceptual quality evaluation for image defocus deblurring,” Signal Processing: Image Communication, vol. 48, pp. 81-91, 2016. Article (CrossRef Link).

[13] L. D. Li, W. H. Xia, W. S. Lin, Y. M. Fang, and S. Q. Wang, "No-Reference and Robust Image Sharpness Evaluation Based on Multiscale Spatial and Spectral Features," IEEE Transactions on Multimedia, vol. 19, no. 5, pp. 1030-1040, 2017. Article (CrossRef Link).

[14] R. Ferzli, and L. J. Karam, "A No-Reference Objective Image Sharpness Metric Based on the Notion of Just Noticeable Blur,” IEEE Transactions on Image Processing, vol. 18, no. 4, pp. 717-728, 2009. Article (CrossRef Link).

[15] K. Bahrami, and A. C. Kot, "A Fast Approach for No-Reference Image Sharpness Assessment Based on Maximum Local Variation,” IEEE Signal Processing Letters, vol. 21, no. 6, pp. 751-755, 2014. Article (CrossRef Link).

[16] L. D. Li, W. S. Lin, X. S. Wang, G. B. Yang, K. Bahrami, and A. C. Kot, "No-Reference Image Blur Assessment Based on Discrete Orthogonal Moments,” IEEE Transactions on Cybernetics, vol. 46, no. 1, pp. 39 - 50, 2016. Article (CrossRef Link).

[17] M. A. Saad, A. C. Bovik, and C. Charrier, "Blind Image Quality Assessment: A Natural Scene Statistics Approach in the DCT Domain,” IEEE Transactions on Image Processing, vol. 21, no. 8, pp. 3339-3352, 2012. Article (CrossRef Link).

[18] Y. Zhang, and D. M. Chandler, "An algorithm for no-reference image quality assessment based on log-derivative statistics of natural scenes," Image Quality and System Performance X, vol. 8653, pp. 1-11, 2013. Article (CrossRef Link).

[19] L. X. Liu, B. Liu, H. Huang, and A. C. Bovik, "No-reference image quality assessment based on spatial and spectral entropies,” Signal Processing Image Communication, vol. 29, no. 8, pp. 856-863, 2014. Article (CrossRef Link).

[20] K. M. He, J Sun, X. O. Tang, "Single image haze removal using dark channel prior,” in Proc. of IEEE Conference on Computer Vision and Pattern Recognition, pp.1956-1963, 20-25 June 2009. Article (CrossRef Link).

[21] J. S. Pan, D. Q. Sun, H. Pfister, and M. H. Yang, "Blind Image Deblurring Using Dark Channel Prior," in Proc. of 2016 IEEE Conference on Computer Vision and Pattern Recognition, pp. 1628-1636, 2016. Article (CrossRef Link).

[22] B. T. Itu-R, "Methodology for the subjective assessment of the quality of television pictures," Recommendation, International Telecommunication Union/ITU Radiocommunication Sector, 2009.

[23] S. Z. Wang, K. Jin, H. T. Lu, C. Cheng, J. Ye and D. H. Qian, "Human visual system-based fundus image quality assessment of portable fundus camera photographs," IEEE Transactions on Medical Imaging, vol. 35, no. 4, pp. 1046-1055, 2015. Article (CrossRef Link).

[24] Z. Farbman, R. Fattal, and D. Lischinski, "Edge-preserving decompositions for multi-scale tone and detail manipulation,” Acm Siggraph, vol. 27, no. 3, pp.1-10, 2008. Article (CrossRef Link).

[25] Y. B. Zhan and R. Zhang, "No-Reference Image Sharpness Assessment Based on Maximum Gradient and Variability of Gradients,” IEEE Transactions on Multimedia, vol. 20, no. 7, pp. 1796-1808, 2018. Article (CrossRef Link).

[26] Z. Wang and Q. Li, "Information content weighting for perceptual image quality assessment," IEEE Transactions Image Process., vol. 20, no. 5, pp. 1185-1198, 2011. Article (CrossRef Link).

[27] Final Report From the Video Quality Experts Group on the Validation of Objective Quality Metrics for Video Quality Assessment, 2003. 
[28] N. D. Narvekar, and L. J. Karam, “A No-Reference Image Blur Metric Based on the Cumulative Probability of Blur Detection,” IEEE Transactions on Image Processing, vol. 20, no. 9, pp. 2678-2683, 2011. Article (CrossRef Link).

[29] P. Marziliano, F. Dufaux, S. Winkler, and T. Ebrahimi, “A no-reference perceptual blur metric," in Proc. of International Conference on Image Processing, pp. III-57 - III-60, 22-25 Sept. 2002. Article (CrossRef Link).

[30] R. Hassen, Z. Wang, and M. M. A. Salama, "Image Sharpness Assessment Based on Local Phase Coherence,” IEEE Transactions on Image Processing, vol. 22, no. 7, pp. 2798-2810, 2013. Article (CrossRef Link).

[31] C. T. Vu, T. D. Phan, and D. M. Chandler, “A spectral and spatial measure of local perceived sharpness in natural images," IEEE Transactions on Image Processing, vol. 21, no. 3, pp.934-945, 2012. Article (CrossRef Link).

[32] L. D. Li, D. Wu, J. J. Wu, H. L. Li, W. S. Lin, and A. C. Kot, "Image Sharpness Assessment by Sparse Representation,” IEEE Transactions on Multimedia, vol. 18, no. 6, pp.1085-1097, 2016. Article (CrossRef Link).

[33] P. V. Vu, and D. M. Chandler, “A Fast Wavelet-Based Algorithm for Global and Local Image Sharpness Estimation,” IEEE Signal Processing Letters, vol. 19, no. 7, pp. 423-426, 2012. Article (CrossRef Link).

[34] K. Gu, G. T. Zhai, W. S. Lin, X. K. Yang, and W. J. Zhang, "No-reference image sharpness assessment in autoregressive parameter space," IEEE Transactions on Image Processing, vol. 24, no. 10, pp. 3218-3231, 2015. Article (CrossRef Link).

[35] Q. B. Sang, H. X. Qi, X. J. Wu, C. F. Li, and A. C. Bovik, "No-reference image blur index based on singular value curve,” Journal of Visual Communication \& Image Representation, vol. 25, no. 7, pp. 1625-1630, 2014. Article (CrossRef Link).

[36] A. Mittal, A. K. Moorthy, and A. C. Bovik, "No-reference image quality assessment in the spatial domain,” IEEE Transactions on Image Processing, vol. 21, no. 12, pp. 4695-4708, 2012. Article (CrossRef Link).

[37] A. K. Moorthy, and A. C. Bovik, “A Two-Step Framework for Constructing Blind Image Quality Indices,” IEEE Signal Processing Letters, vol. 17, no. 5, pp. 513-516, 2010. Article (CrossRef Link).

[38] A. K. Moorthy, and A. C. Bovik, "Blind Image Quality Assessment: From Natural Scene Statistics to Perceptual Quality,” IEEE Transactions on Image Processing, vol. 20, no. 12, pp. 3350-3364, 2011. Article (CrossRef Link).

[39] A. Mittal, R. Soundararajan, and A. C. Bovik, "Making a "completely blind” image quality analyzer,” IEEE Signal Processing Letters, vol. 20, no. 3, pp. 209-212, 2013. Article (CrossRef Link).

[40] W. F. Xue, L. Zhang, and X. Q. Mou, "Learning without human scores for blind image quality assessment," in Proc. of IEEE Conference on Computer Vision and Pattern Recognition, pp. 995-1002, 23-28 June 2013. Article (CrossRef Link).

[41] D. M. Chandler and S. S. Hemami, "VSNR: A wavelet-based visual signal-to-noise ratio for natural images,” IEEE Transactions on Image Processing, vol. 16, no. 9, pp. 2284-2298, 2007. Article (CrossRef Link). 


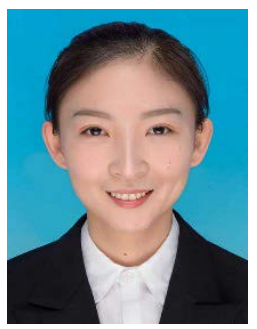

Qiaoyue Li received the B.S. degree in Information and Communication Engineering from China University of Mining and Technology in 2012. She is currently working toward the M.S. Degree in the School of Information and Control Engineering, China University of Mining and Technology. Her research interests include image quality assessment and perceptual image processing.

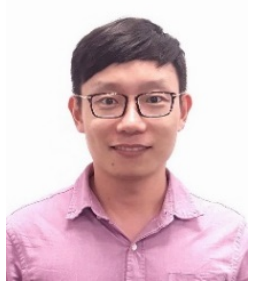

Leida Li received the B.S. and Ph.D. degrees from Xidian University, Xi'an, China, in 2004 and 2009, respectively. From February to June 2008, he was a visiting Ph.D. student in the Department of Electronic Engineering, National Kaohsiung University of Applied Sciences, Taiwan. From January 2014 to January 2015, he was a Visiting Research Fellow in the Rapid-Rich Object Search (ROSE) Laboratory, School of Electrical and Electronic Engineering, Nanyang Technological University, Singapore. From July 2016 to July 2017, he was a Senior Research Fellow in the same lab, Nanyang Technological University, Singapore. Currently, he is a Full Professor in the School of Information and Control Engineering, China University of Mining and Technology, China. His research interests include multimedia quality assessment, information hiding and image forensics.

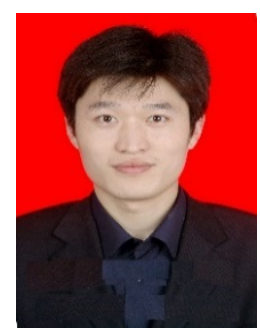

Zhaolin Lu is an associate professor in Advanced Analysis \& Computation Center, China University of Mining and Technology. He received the Ph.D. degree in computer application technology from China University of Mining and Technology in 2012. His current research interests include microscopic mineral image quality evaluation, mineral particle recognition and image analysis of mineral occurrence pattern in coal.

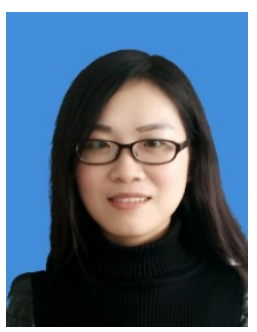

Yu Zhou received the B.S. degree from China University of Mining and Technology, Xuzhou, China, in 2014. She is now pursuing the Ph.D. degree in the School of Information and Control Engineering, China University of Mining and Technology, China. Her research interests include multimedia quality assessment and perceptual image processing.

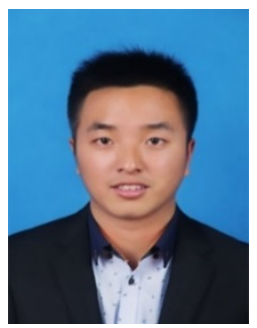

Hancheng Zhu received the B.S. degree in Electronic Information Engineering from Changzhou Institute of Technology in 2012. He received the M.S. degree in Information and Communication Engineering from China University of Mining Technology in 2015. He is currently working toward the PH.D. degree at the School of Information and Control Engineering, China University of Mining Technology. His research interests include deep learning, affective computing, and image aesthetic assessment. 